\title{
ERK2 and Akt are negative regulators of insulin and Tumor Necrosis Factor-a stimulated VCAM- 1 expression in rat aorta endothelial cells
}

\author{
Gregory B. Pott ${ }^{1}$, Mark Tsurudome ${ }^{1}$, Nadia Bamfo ${ }^{1}$ and Marc L. Goalstone ${ }^{1,2^{*}}$
}

\begin{abstract}
Background: Diabetes is quickly becoming the most widespread disorder in the Western world. Among the most prevalent effects of diabetes is atherosclerosis, which in turn is driven in part by inflammation. Both insulin and Tumor Necrosis Factor-alpha (TNFa) increase the presence of Vascular Cellular Adhesion Molecule-1 (VCAM-1) expression. The aim of this study is to determine the effects of downregulating Extracellular signal-Regulated Kinase-2 (ERK2) and Akt on insulin and TNFa-stimulated VCAM-1 expression.

Methods: Here we begin to define the relationships between ERK2 and Akt regulation of insulin and TNFa-stimulated VCAM-1 expression in Rat Arterial Endothelial Cells (RAEC) by transfecting RAEC with ERK2 and Akt RNA interference (RNAi) and then treating these cells with insulin $(10 \mathrm{nM})$ or TNFa $(10 \mathrm{ng} / \mathrm{mL})$ alone or in combination.

Results: Western blot analyses, flow cytometry and confocal microscopy were used to determine changes in VCAM-1 expression within the above-stated parameters. Cells transfected with ERK2 or Akt RNAi plasmids increased insulin and TNFa-stimulated VCAM-1 total protein expression significantly $(P<0.05)$ greater than that seen in mock transfected cells and expressed cell surface VCAM-1 greater than that seen in mock transfected cells as indicated by flow cytometry and confocal microscopy. Nevertheless, the decrease of both kinases did not increase insulin or TNFa-stimulated VCAM-1 expression above that seen when one or the other RNAi was present.

Conclusions: Taken together, our results demonstrate that ERK2 and Akt may be negative regulators of insulin and TNF-a stimulated VCAM-1 and that their loss or down regulation might upregulate VCAM-1 expression and contribute to vascular disease.
\end{abstract}

Keywords: Atherosclerosis, Inflammation, ERK2, Akt, RNAi, Insulin, TNFa, VCAM-1

\section{Background}

Diabetes mellitus (DM) is a serious global health problem. In 2014, 29.1 million adults were clinically diagnosed with diabetes and $\$ 245$ billion dollars were spent on the treatment for diabetes in the United States (US) alone. Diabetes brings along with it a sequela of adverse conditions such as, but not limited to, insulin resistance, dyslipidemia, retinopathy, nephropathy, and

\footnotetext{
* Correspondence: Marc.Goalstone@va.gov

'Division of Endocrinology, Metabolism and Diabetes, University of Colorado Anschutz Medical Campus, 12801 East 17th Avenue. Mail Stop: 8106, Aurora, CO 80045, USA

${ }^{2}$ Eastern Colorado Health Care System, (Denver VA Medical Center), 1055 Clermont Street. Mail Stop 151, Denver, CO 80220, USA
}

cardiovascular disease (CVD) [1]. CVD is among the most aggressive aspects of DM and is associated with plaque formation, decreased blood flow rate, thrombosis, occlusion and rupture of the arteries, morbidity and mortality [2].

Atherosclerosis has been linked to a range of cellular and molecular changes in the vascular wall most of which are noted in the endothelial cells of the arteries [3]. Associated with the remodeling of the arterial walls is the expression of the endothelial surface molecule, Vascular Cell Adhesion Molecule-1 (VCAM-1) [4]. VCAM-1 appears to be upregulated in DM patients' endothelial cells. The increase of VCAM-1 appears to 
foster the recruitment of monocytes to the surface of the endothelium and their subsequent transmigration across the endothelial layer [5]. Once embedded into the vascular smooth muscle layer, these monocytes mature to fully functional macrophages, which subsequently activate and express inflammatory cytokines such as Tumor Necrosis Factor-alpha (TNF $\alpha$ ) [4].

Hyperinsulinemia (HI) and increased presence of TNF $\alpha$ are consequences of insulin resistance $[1,6]$. Both $\mathrm{HI}$ and TNF $\alpha$ in turn exacerbate the pathophysiological conditions of the endothelium and cause an increase in VCAM-1 expression. Expression of VCAM-1 is regulated by a family of kinases, which mediate external signals to internal events $[7,8]$. These kinases include, but are not limited to, extracellular signal-regulated kinase 1/2 (ERK1/2), protein kinase B/Akt (Akt), p38 kinase and c-Jun N-terminal kinase (JNK). ERK2 and Akt are major mediators of external signals to internal events. Others have shown that Akt and ERK2 contribute to the regulation of VCAM-1 [9, 10]. Thus, we started our studies examining these two kinases and their effects on VCAM-1 expression. Here we report that the reduction of ERK2 or Akt in rat aorta endothelial cells (RAEC) increases insulin and TNF $\alpha$-stimulated total VCAM-1 expression. In contrast, the simultaneous reduction of both ERK2 and Akt did not cause an additive increase in VCAM-1 protein above that of ERK2 or Akt alone. Although there may not be a cumulative effect of increased VCAM-1 due to the decreased presence of ERK2 and Akt in endothelial cells, their diminished presence may play a significant role in the inflammatory attributes of cardiovascular disease.

Here we report that when the expression of ERK2 and Akt was decreased via RNA interference, we observed insulin and TNFo-stimulated increased VCAM-1 expression at the protein and cell surface level. However, simultaneous downregulation of both ERK2 and Akt did not show an additive or synergistic effect.

\section{Methods}

Materials

All general lab reagents were purchased from SigmaAldrich (St. Louis, MO). PVDF membranes and other Western blot accessories were from GE Healthcare/ Amersham (Piscataway, NJ). Primary rabbit antibodies to ERK1/2 (9102), Akt (4056), and alpha-tubulin (2144S) were from Cell Signaling (Boston, MA). The primary rabbit antibody to VCAM-1 (NBP1-95622) was from Novus Biologicals (Littleton, CO) and goat anti-rabbitsecondary antibody IRDye680RD (926-68171) was from LI-COR (Lincoln, NE). Rat aorta vascular endothelial cells (RAEC) (CRL-1444) were from ATCC (Manassas, VA) and culture medium was from Life Technologies (Grand Island, NY). ERK2 (KR48780P) and Akt (KR45425P) short hairpin (sh) RNA plasmids were obtained from SA Biosciences/Qiagen (Valencia, CA). Transfection Medium (108062) and Reagent ((108061) were from Santa Cruz Biotechnology (Dallas, Tx). DyLight 488-conjugated anti-VCAM-1 antibody was from Thermo Scientific (Pittsburgh, PA). Four-well chamber slides were from Thermo Fisher and DAPI Mounting Medium was from Vector Labs (Burlingame, CA). Insulin (19278) was from Sigma-Aldrich (St. Louis, MO) and TNFa (11271156001) was from Roche (Indianapolis, IN).

\section{Cell culturing}

RAEC were cultured in complete growth medium (CGM) [DMEM with $4 \mathrm{mM}$ L-glutamine, $4.5 \mathrm{~g} / \mathrm{L}$-glucose and $1.5 \mathrm{~g} / \mathrm{L}$ sodium bicarbonate) and supplemented with $10 \%$ heat-inactivated fetal bovine serum (HI-FBS) (10438-026) (Life Technologies, Grand Island, NY) and $1 \%$ Antimycotic-Antibiotic solution (15240-062) (Life Technologies) and cultured at $37{ }^{\circ} \mathrm{C}, \quad 5 \% \mathrm{CO}_{2}$ atmosphere.

\section{Preparation of shRNA stable cell lines}

RAEC were grown to 50-70 \% confluence in CGM in 6-well culture plates. Cells were transfected with shERK2 (clone \#2) or shAkt (clone \#2) inhibitory plasmids as previously described [11]. Cells were incubated in CGM containing $2 \mu \mathrm{g} / \mathrm{mL}$ of Puromycin (Sigma-Aldrich) for 2-3 weeks for selection of Puromycin resistant transformants.

\section{Dual transfection of stable cell lines}

To examine the effect of simultaneous ERK2 and Akt knockdown on VCAM-1 expression, the ERK2 shRNA stable cell line (ERK2 KD) was transiently transfected with shAkt plasmid and the Akt shRNA stable cell line (Akt KD) was transiently transfected with the shERK2 plasmid. These two protocols were carried out in order to see if any difference occurred with respect to transfection sequence. Stable cell lines were transiently transfected with shRNA plasmid DNA as described above and incubated for $5 \mathrm{~h}$ with the DNA transfection mix. Subsequently the transfection mix was aspirated and replaced with $2.0 \mathrm{~mL}$ CGM. Stimulation of cells by insulin and/or TNF $\alpha$ occurred $48 \mathrm{~h}$ after transient transfection was accomplished.

\section{Stimulation of VCAM-1 expression}

RAEC were cultured in CGM, whereas shRNA stable cell lines (e.g., ERK2 KD and Akt KD) were cultured in CGM containing $2 \mu \mathrm{g} / \mathrm{mL}$ Puromycin until assays were performed. After incubating the transfected cells for an additional $48 \mathrm{~h}$, the cells were stimulated with or without insulin $(10 \mathrm{nM})$ and in the presence or absence of TNF $\alpha$ $(10 \mathrm{ng} / \mathrm{mL})$ and evaluated for VCAM-1 expression as 
previously described [11]. Briefly, TNF $\alpha$ stimulation occurred over a total of $6 \mathrm{~h}$ and cells activated with insulin were stimulated for $1 \mathrm{~h}$.

\section{Western blot analysis}

Sodium Dodecyl Sulfate Polyacrylamide Electrophoresis was performed on cleared lysates. Western blot analysis was subsequently performed as previously described, [11] with the following differences. After completion of protein transfer, membranes were washed in ultra-pure water for $5 \mathrm{~min}$. Membranes were then incubated in $3 \%$ non-fat milk (milk) in Tris-buffered Saline (TBS) blocking solution for $1 \mathrm{~h}$ at room temperature and then incubated with a designated primary antibody solution (1:1000 in $3 \%$ milk/TBS-T) overnight at $4{ }^{\circ} \mathrm{C}$. Membranes were washed 4 times with TBS plus Tween (TBS-T) for $5 \mathrm{~min}$ at room temperature and then incubated with a goat anti-rabbit secondary antibody (1:5000 in $3 \%$ milk/TBS-T) conjugated to fluorochrome IR680RD for $1 \mathrm{~h}$ at room temperature. Membranes were washed 4 times with TBS-T for $5 \mathrm{~min}$ at room temperature and then incubated with a rabbit anti-tubulin primary antibody solution (1:1000 in $3 \%$ milk/TBS-T) for $3 \mathrm{~h}$ at room temperature. After washing the membranes for 4 times with TBS-T, the membranes were again incubated with a goat anti-rabbit secondary antibody (1:5000 in $3 \%$ milk/TBS-T) conjugated to fluorochrome IR680RD for $1 \mathrm{~h}$ at room temperature. The membranes were washed 4 times with TBS-T and allowed to dry before performing densitometry. Densitometry was performed using an Odyssey Licor system (Lincoln, NE). Alphatubulin protein was used to normalize VCAM-1 signals.

\section{Flow cytometry}

Non-transfected RAEC were used as controls. Stably transfected RAEC Akt knockdown (Akt KD) and ERK2 knockdown (ERK2 KD) cell lines were inoculated into 6well tissue culture dishes, transiently transfected with shERK2 or shAkt plasmids, respectively, and stimulated with insulin, TNF $\alpha$, or combined insulin and TNF $\alpha$ as described above in Dual Transfection. The cells were washed twice with $2 \mathrm{~mL}$ of $1 \mathrm{X}$ PBS (Gibco). The PBS was aspirated and $0.5 \mathrm{~mL}$ of Cell Dissociation Solution Non-Enzymatic (Sigma-Aldrich) was added to each well. After incubating the cells at $37{ }^{\circ} \mathrm{C}$ and $5 \% \mathrm{CO}_{2}$ for $30 \mathrm{~min}, 1 \mathrm{~mL}$ of $1 \%$ Bovine Serum Albumin (BSA, Sigma-Aldrich) in PBS was added to the cells and then were gently triturated into a single cell suspension. The cells were transferred to $5 \mathrm{~mL}$ Falcon polystyrene round bottom tubes (Thermo Scientific) and centrifuged at 500 $\mathrm{x} g$ for $5 \mathrm{~min}$. After aspirating the supernatants, the cells were resuspended in $3 \mathrm{~mL} 1 \% \mathrm{BSA}$, pelleted at $500 \times \mathrm{g}$ by centrifugation, and the supernatants removed by aspiration. The cells were resuspended in $200 \mu \mathrm{L}$ of $1 \%$
BSA. Two microliters of DyLight 488-conjugated antiVCAM-1 antibody (Life Technologies, Grand Island New York) were added to each tube and the cells were resuspended by vortexing. The cells were incubated in the dark for $30 \mathrm{~min}$ at room temperature. The cells were centrifuged, washed twice with $3 \mathrm{~mL} 1 \%$ BSA and resuspended in $200 \mu \mathrm{L}$ of $1 \%$ paraformaldehyde (PFA, Electron Microscopy Sciences, Hatfield, PA). After incubating the cells for $5 \mathrm{~min}$ at room temperature, the cells were diluted with an additional $300 \mu \mathrm{L}$ of PBS and analyzed using flow cytometry.

The experiments were run on a BD LSRII (BD Biosciences, San Jose, CA). MFI and gating percentages as part of data analysis were performed using BD FACSDiva v6 software.

\section{Chamberslide cell preparation}

$2 \times 10^{5}$ of control RAEC, Akt KD or ERK2 KD stable cell lines were plated in $1 \mathrm{~mL}$ of CGM in each well of a 4-well chamberslide and allowed to grow for $24 \mathrm{~h}, 37^{\circ} \mathrm{C}$ and $5 \% \mathrm{CO}_{2}$. The medium was aspirated and $1.0 \mathrm{~mL}$ of fresh CGM was applied to the cells. Akt KD and ERK2 KD were either mock transfected, or transiently

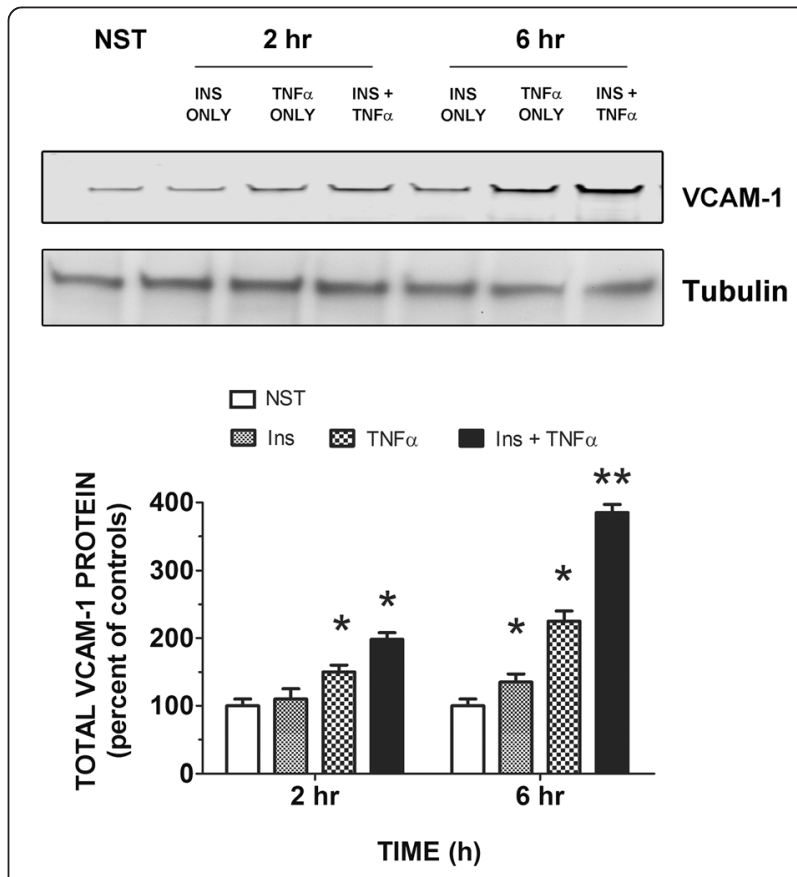

Fig. 1 Insulin and TNFa increase expression of total VCAM-1 in rat aorta endothelial cells over time. RAEC were cultured in Complete Growth Medium (CGM) until $90 \%$ confluent. Subsequently, cells were stimulated for indicated times with insulin (10 nM) or TNFa $(10 \mathrm{ng} / \mathrm{mL})$ alone or in combination. Total VCAM-1 protein was determined by Western blot analysis. Analysis was performed by the Licor Odyssey detection system. Total VCAM-1 is expressed as the percent of controls and represents the mean \pm SEM of six independent experiments. ${ }^{*}, P<0.05$ vs negative controls. ${ }^{* *}, P<0.05$ vs TNFa alone 
transfected with shERK2 or shAkt, respectively for $48 \mathrm{~h}$. Cells were then treated with either vehicle, $10 \mathrm{ng} / \mathrm{mL}$ $\mathrm{TNF} \alpha$, insulin $(10 \mathrm{nM})$ alone, or TNF $\alpha(10 \mathrm{ng} / \mathrm{mL})$ and then insulin $(10 \mathrm{nM})$. The medium was aspirated and the cells were washed three times with PBS and then incubated in $400 \mu \mathrm{L}$ of $4 \%$ paraformaldehyde in PBS for $30 \mathrm{~min}$. The medium was aspirated and washed three times with $1 \mathrm{~mL}$ of PBS. The final PBS wash was aspirated and $400 \mu \mathrm{L}$ of a 1:1000 DyLight anti-VCAM-1 antibody soluton in $1 \%$ BSA was added to each chamber and incubated for $30 \mathrm{~min}$ at room temperature. The cells were then washed three times with $500 \mu \mathrm{L}$ of $1 \%$ BSA. The chamber walls were removed and one drop of DAPI Mounting Medium was added to each group of cells on the slide. Cells were then sealed with a glass

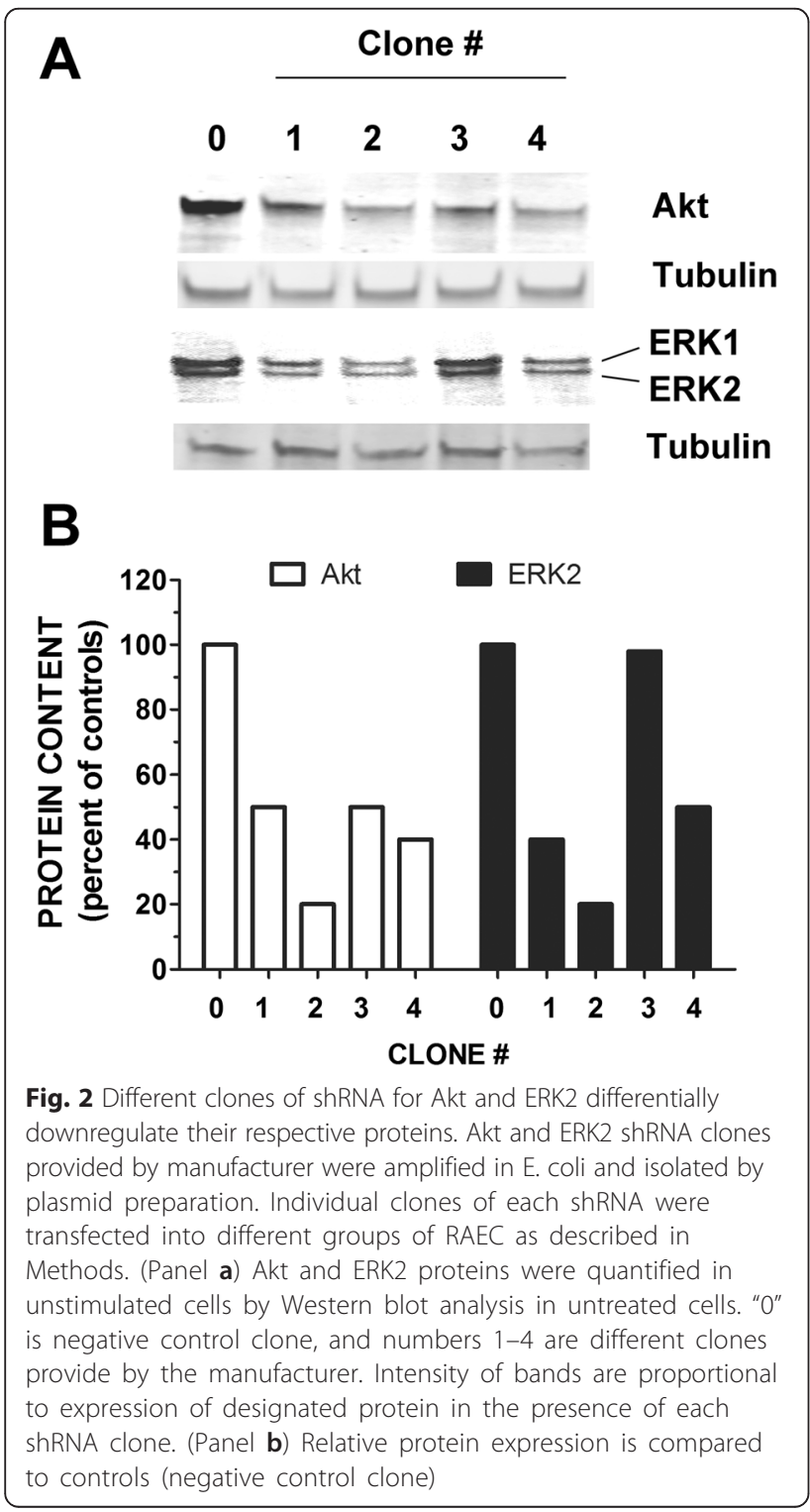

cover slip using clear nail polish. Slides were kept in a dark refrigerator until microscopic visualization.

\section{Confocal microscopy}

A single, non-confluent monolayer of cells were imaged with a Leica TSC SP8X white light laser scanning confocal microscope (Leica Microsystems $\mathrm{GmbH}$. Ernst-Leitz-Straße 17-37 Wetzlar, 35578 Germany). Excitation of the DAPI channel was achieved using a $405 \mathrm{~nm}$ diode laser with an excitation intensity level of $2.67 \%$. Emission signal was captured with standard PMT Channel 1 with an emission gap of $430 \mathrm{~nm}$ $480 \mathrm{~nm}$. Excitation of ALexa fluor 488 utilized the Leica Supercontinuum white light laser visible excitation laser line $(488 \mathrm{~nm})$ and an intensity level of $3 \%$. Emission signal was captured with Leica HyD 2 detector (Hybird 2 PMT) with a with a set emission gap of $(505 \mathrm{~nm}-555 \mathrm{~nm})$. All image acquisitions

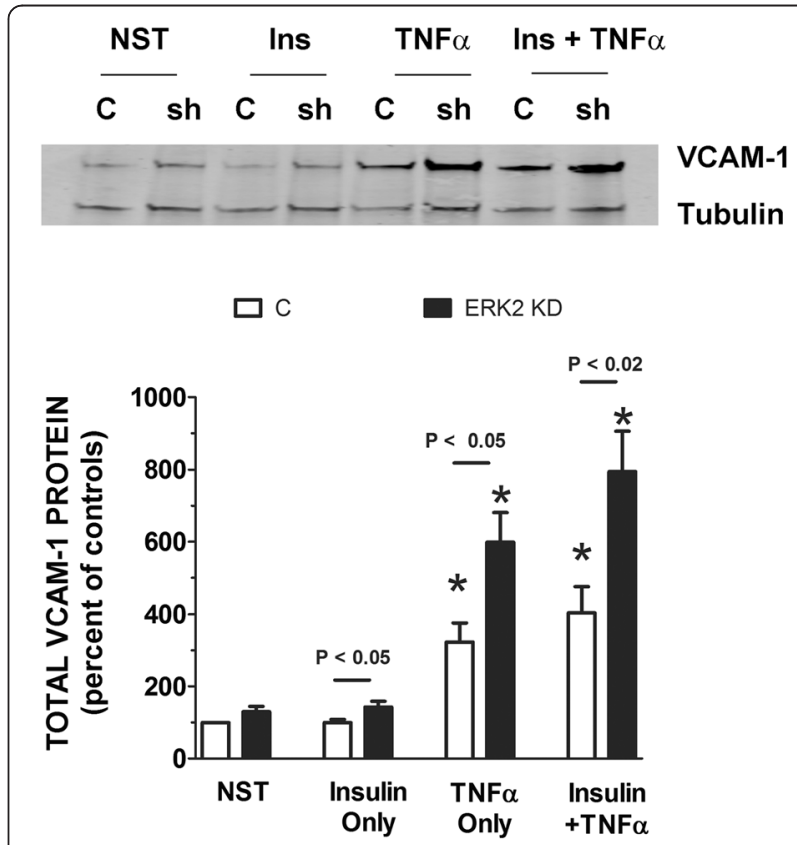

Fig. 3 RAEC transfected with shERK2 (clone \#2) and then stimulated with insulin or TNFa alone or in combination demonstrates the negative regulation of ERK2 in VCAM-1 expression. RAEC were stably transfected with shERK2 (ERK2 KD) and then stimulated with insulin $(10 \mathrm{nM}, 1 \mathrm{~h})$ or TNFa $(10 \mathrm{ng} / \mathrm{mL}, 6 \mathrm{~h})$ alone or in combination. Total VCAM-1 protein was analyzed by SDS-PAGE and determined by Western blot analysis. (Upper Panel) "NST", cells not stimulated with insulin or TNFa. "Ins" cells stimulated with insulin. "TNFa" cells stimulated with TNFa. " $\mathrm{C}$ " control cells not transfected with shRNA, while "sh" cells were transfected with shERK2. (Lower Panel) " $\mathrm{C}$ " control RAEC not transfected (open bars). ERK2 KD are RAEC stably transfected with shERK2 (black bars). "NST" cells not stimulated with insulin or TNFa. Total VCAM-1 is expressed as the percent of controls and represents the mean \pm SEM of six independent experiments. Alpha-tubulin was used to normalize VCAM-1 profiles. ${ }^{*}, P<0.05$ vs non-stimulated cells 
were carried out using the Leica Application Suite X (version 1.1.0.12420, LASX AF).

\section{Data analysis}

Data were analyzed by either unpaired Student's $t$ test (two groups) or ANOVA with subsequent Tukey posttest (several groups) as indicated. A "P" value of less than 0.05 was considered significant. Results were expressed as the mean \pm SEM of three or more independent experiments.

\section{Results}

Both insulin and TNF $\alpha$ significantly $(P<0.05)$ increased VCAM-1 at $6 \mathrm{~h}$ as compared to controls (Fig. 1). TNF $\alpha$ stimulated VCAM-1 was significantly $(P<0.05)$ greater than that seen for insulin alone at 2 and $6 \mathrm{~h}$ time points. Interestingly, cells stimulated with insulin plus TNF $\alpha$ for $6 \mathrm{~h}$ increased VCAM-1 significantly $(P<0.05)$ greater than that seen for insulin or TNF $\alpha$ alone.

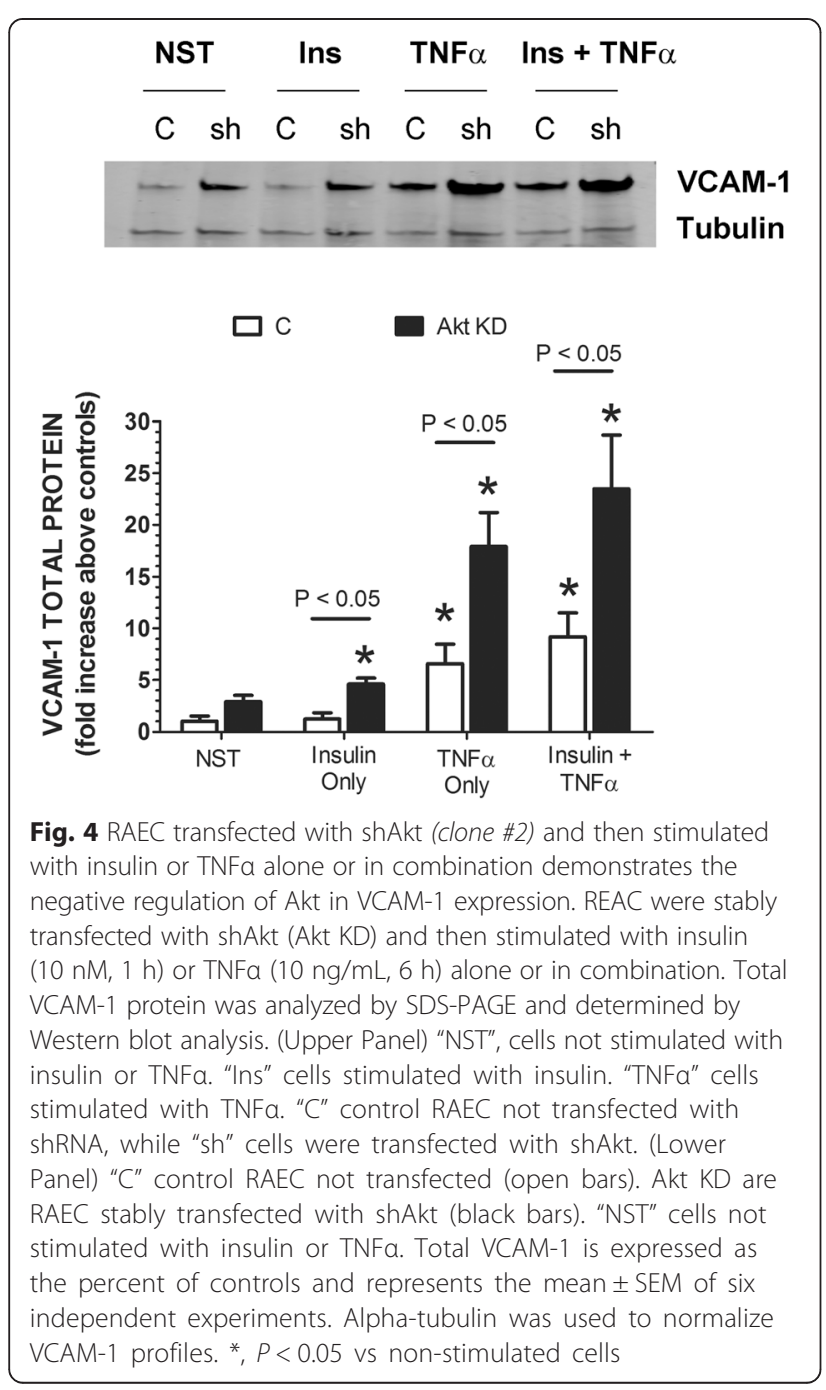

In order to tease out the intracellular kinase regulators of these events, we incorporated RNA interference into these experiments to better understand which kinases were mediating the external signals of insulin and TNF $\alpha$ with regards to the expression of VCAM-1 protein.

In previous studies, we investigated the effect of ERK5 knockdown on insulin and TNF $\alpha$-stimulated VCAM-1 [11]. In those studies, decreased ERK5 resulted in decreased insulin and TNF $\alpha$-stimulated VCAM-1 expression. To continue this line of study we measured the expression of VCAM-1 in vascular cells in which expression of either ERK2 or Akt or both were decreased using RNAi techniques and in the presence of insulin and/or TNF $\alpha$.

After first determining which shERK2 and shAkt plasmid clone decreased its respective kinase most significantly (Fig. 2), we transfected the most efficacious shERK2 plasmid clone $(\# 2)$ into our cells and treated with either insulin $(10 \mathrm{nM})$ or TNF $\alpha(10 \mathrm{ng} / \mathrm{mL})$ alone

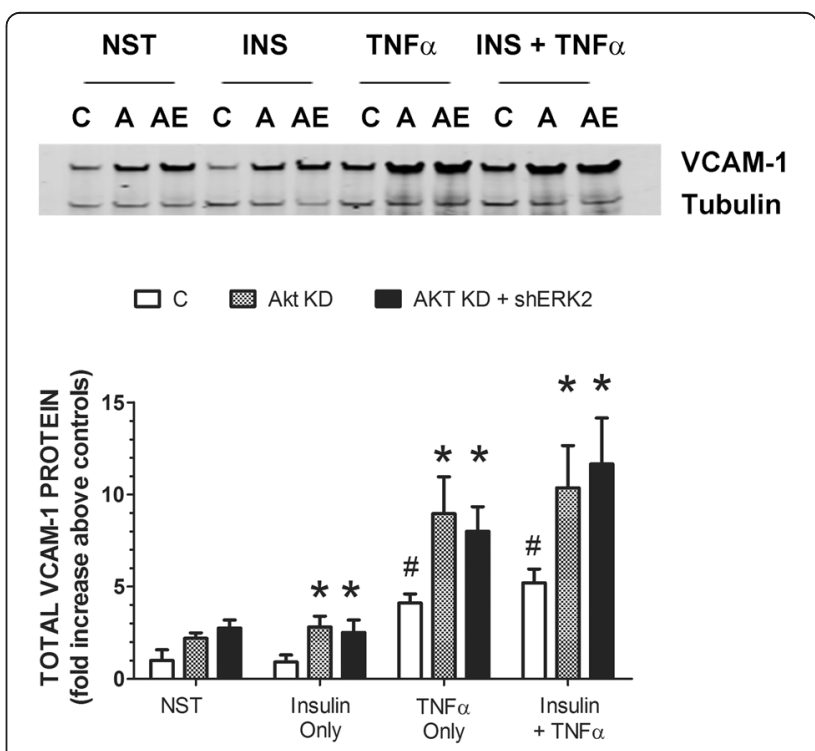

Fig. 5 RAEC transfected with shAkt and shERK2 and then stimulated with insulin or TNFa alone or in combination exhibit increased VCAM-1 protein expression greater than stimulated, mock transfected cells, but not greater than cells transfected with shAkt alone. RAEC were stably transfected with shAKT and then transiently transfected with shERK2. Subsequently, cells were stimulated with insulin (10 nM, $1 \mathrm{~h})$ or TNFa $(10 \mathrm{ng} / \mathrm{mL}, 6 \mathrm{~h})$ or in combination. (Upper Panel) C, control RAEC, no shRNA. A, cells transfected with shAkt alone. AE, cells stably transfected with shAkt and then transiently transfected with shERK2. (Lower Panel) Open bars are control cells not transfected. Stippled bars (Akt KD) RAEC stably transfected with shAkt. Black bars (Akt KD + shERK2) RAEC stably transfected with shAkt and transiently transfected with shERK2. "NST", cells not stimulated with insulin or TNFa. Total VCAM-1 is expressed as fold increase above negative controls and represents the mean \pm SEM of five independent experiments. Alpha-tubulin was used to normalize VCAM-1 profiles. \#, $P<0.05$ verses non-stimulated controls. ${ }^{*}, P<0.05$ versus stimulated controls of similar treatment group without shRNA 
or in combination for designated times. Subsequently, we measured the change in total VCAM-1 protein between positive controls (no shRNA) and ERK2 KD cells. Interestingly, in the ERK2 KD cells, insulin-stimulated VCAM-1 increased $52 \%$ above positive controls (cells treated with insulin with no shERK2) and TNF $\alpha$-alone stimulated VCAM-1 84 \% above positive controls (TNF $\alpha$ - stimulated cells with no shERK2) (Fig. 3). In ERK2 KD cells, VCAM-1 continued to increase $96 \%$ above combined insulin plus TNF $\alpha$-stimulated positive controls.

We next determined whether shAkt affected insulin and TNF $\alpha$-stimulated VCAM-1 expression. Similar to the results observed with ERK2 cells, the presence of shAkt (Akt KD) significantly increased VCAM-1 in the presence of insulin or TNF $\alpha$ alone 3.8-fold and 2.7-fold, respectively, above positive controls (cells not transfected

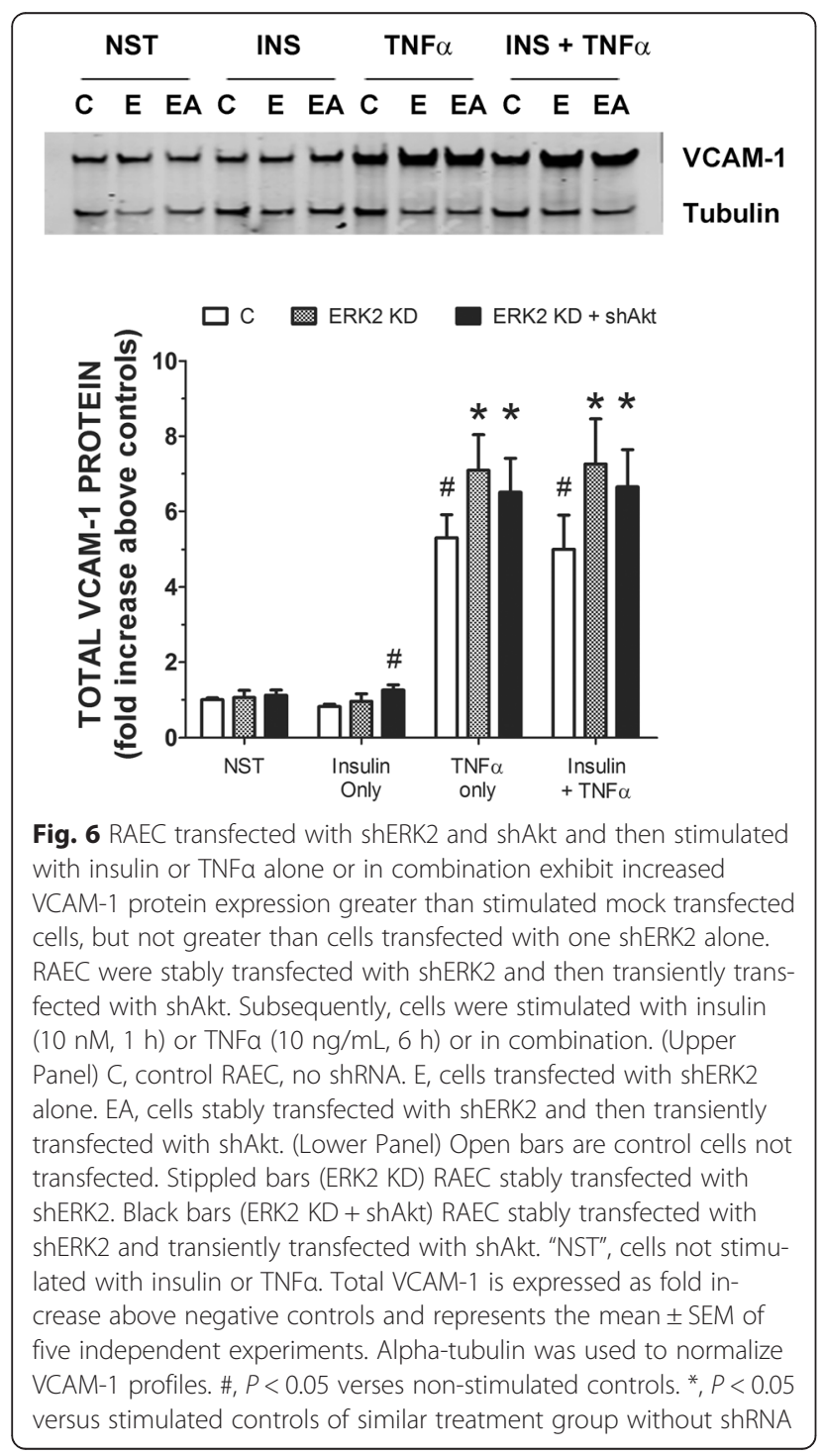

with shRNA) (Fig. 4). In the presence of combined insulin and TNF $\alpha$, Akt KD cells exhibited a 2.6-fold increase in VCAM-1 above positive controls cells treated with insulin and TNFa.

Next, we were interested in changes in VCAM-1 expression in cells stimulated with insulin and/or TNF $\alpha$ and double transfected with shAkt and then shERK2. First, RAEC were stably transfected with shAkt (2 weeks before treatment) and then transiently transfected with shERK2 $48 \mathrm{~h}$ before insulin and TNF $\alpha$ treatment. Interestingly, Akt KD cells transiently transfected with

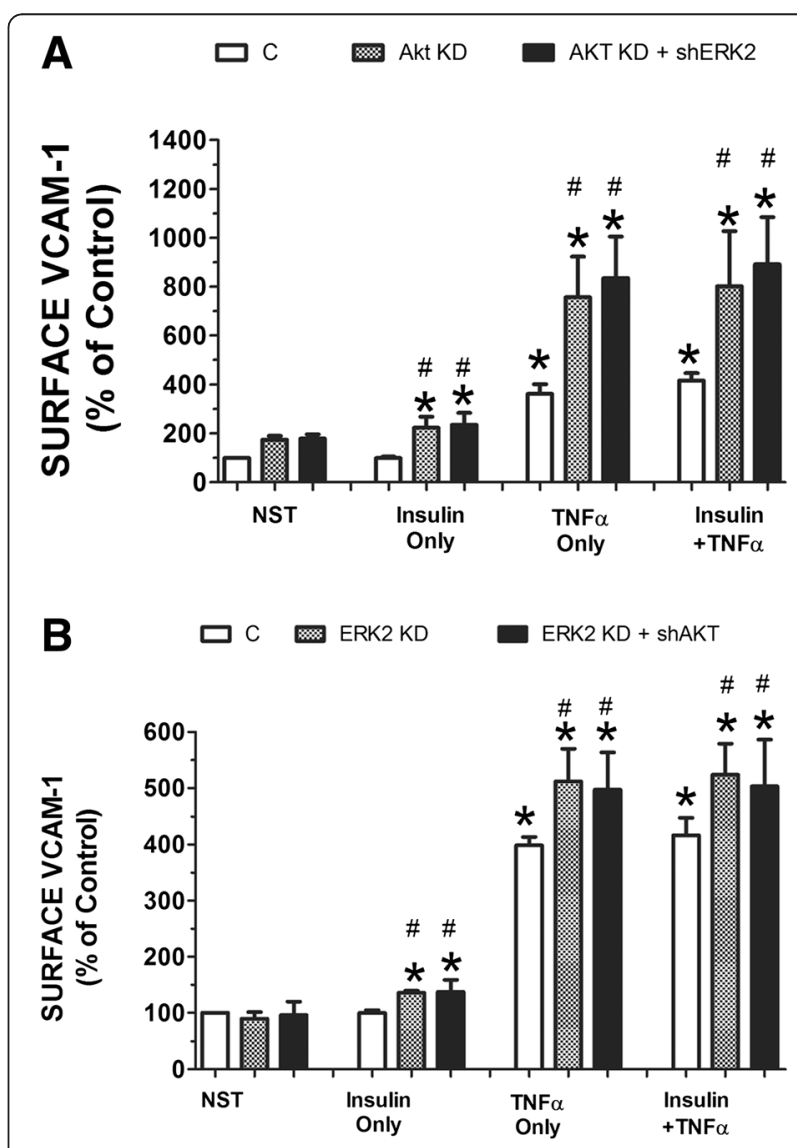

Fig. 7 RAEC transfected with shAkt and shERK2 exhibit increased insulin and TNFa-stimulated VCAM-1 at the cell surface as determined by flow cytometery. (Panel a) RAEC were stably transfected with shAkt (Akt KD) or (Panel b) shERK2 (ERK2 KD) and then transiently transfected with shERK2 or shAkt, respectively. Subsequently, cells were stimulated without or with insulin $(10 \mathrm{nM})$ or TNFa $(10 \mathrm{ng} / \mathrm{mL})$ or in combination and surface VCAM- 1 was determined by flow cytometry. C, non-transfected RAEC (no shRNA). NST, no stimulation. Akt KD, stable cell lines with shAkt. ERK2 KD, stable cell lines with shERK2. Akt $\mathrm{KD}+$ shERK2, stable shAkt cells lines transiently transfected with shERK2. ERK2 KD + shAkt, stable cell lines with shERK2 transiently transfected with shAkt. Surface VCAM-1 is expressed as percent of respective controls (not stimulated, No Stim) and represents the mean \pm SEM of five independent experiments. ${ }^{*}, P<0.05$ compared to their respective unstimulated controls. \#, $P<0.05$ compared to stimulated non-transfected controls 
shERK2 exhibited no increases in insulin or TNFo stimulated VCAM-1 significantly greater than that seen in Akt KD cells alone (Fig. 5).

We wanted to see if altering the sequence of transfected shRNA would affect the outcome of VCAM-1 expression. In experiments similar to those depicted in Fig. 5, we stably transfected RAEC with shERK2, waited 2 weeks and then transiently transfected the cells with shAkt. Similar to the experiments in which shAkt was stably expressed, ERK2 KD expressing transiently transfected shAkt and stimulated with either insulin or TNF $\alpha$ alone or in combination did exhibit increased VCAM-1 above positive controls (no shRNA) (Fig. 6). However, similar to that observed in Fig. 5, expression of VCAM1 was not significantly greater in ERK2 KD cells transiently transfected with shAkt as compared to ERK2 KD cells alone.

Finally, we wanted to determine whether increased VCAM-1 at the total cellular protein level equated to increases at the cell surface. We used two different procedures to determine changes in VCAM-1 at the cell surface: flow cytometry and confocal microscopy. Using flow cytometry, both the Akt KD and ERK2 KD cell lines exibited significantly $(P<0.05)$ greater expression of VCAM-1 at the surface in the presence of insulin and/or TNF $\alpha$ than cells mock transfected (Fig. 7). In order to visualize the increases in VCAM-1 at the cell surface, we utilized confocal microscopy and again observed increased VCAM-1 at the cell surface in Akt KD and ERK2 KD cell lines (Fig. 8).

\section{Discussion}

DM is a pervasive disease that affects old and young alike, and is followed by a sequala of effects. Cardiovascular disease and atherosclerosis are major players in the insidious repertoire of DM and their existence is based upon increased amounts of, but not limited to, serum insulin and TNFa. In turn, these biomoleculer signals escalate downstream cellular events such as increased expression of VCAM-1. Equally important are the kinase mediators that transduce the serum and cell surface signals of insulin and TNF $\alpha$ to intracellular events. ERK2 and Akt are members of this large kinase family and although they may not be the only intracellular mediators of these external signals, they appear to be conduits of inflammatory regulation within endothelial cells.

Kinases such as ERK2 and Akt are major players in intracellular signaling [12]. Down regulation of one may have profound effects in intracellular events [13-16]. Loss of positive effectors or negative inhibitors may cause unknown effects in the cell and the vasculature. The loss of positive players may cause decreases in positive-regulation of key pathways, thereby decreasing their beneficial effects. Alternatively, the loss of negative effectors (i.e., inhibitors) may cause the loss of negativeregulation in the same or parallel pathways. In the

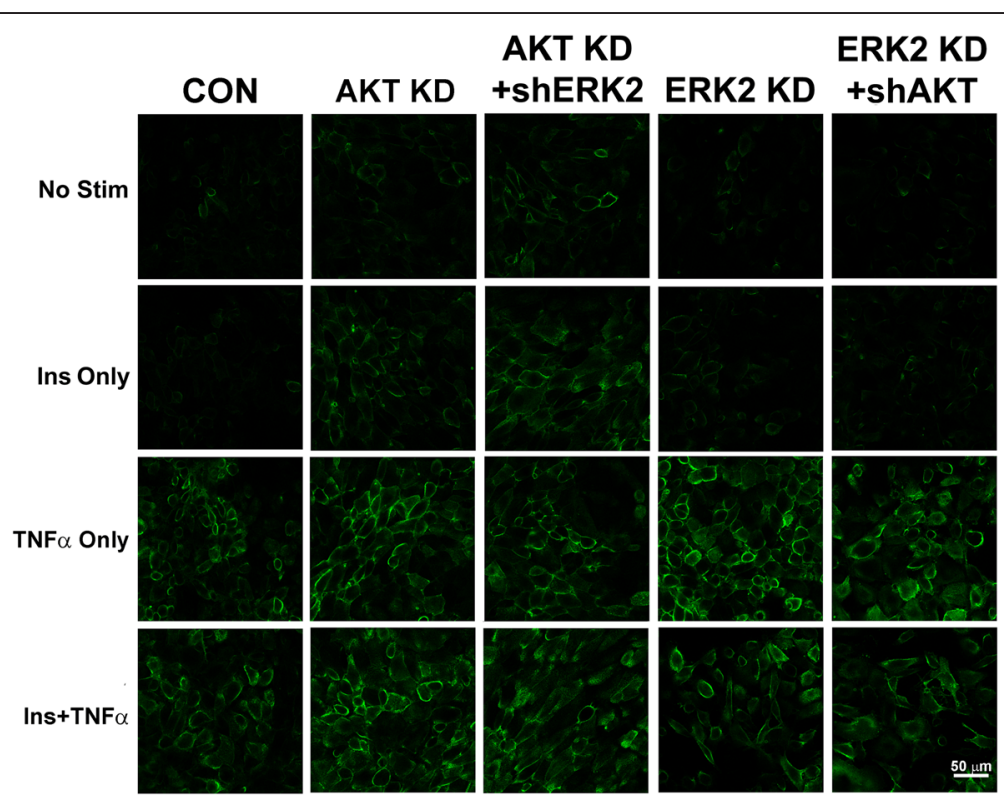

Fig. 8 RAEC transfected with shAkt and shERK2 exhibit increased insulin and TNFa-stimulated cell surface VCAM-1 as determined by confocal microscopy. RAEC were plated and stimulated as described in Methods. Surface VCAM-1 was detected by immunocytochemistry as described in Methods and visualized by confocal microscopy. CON, RAEC controls, no shRNA; No Stim, no stimulation by insulin or TNFa; Akt KD, stable cell lines transfected with shAkt. ERK2 KD, stable cell lines transfected with shERK2. shAkt, cells transiently transfected with shAkt. shERK2, cells transiently transfected with shERK2. Scale bar is $50 \mu \mathrm{m}$ 
current report, knock-down of ERK2 and Akt appeared to perturb the physiologic (inhibitory) qualitiy of these kinases, eliciting an upregulation of VCAM-1. Interestingly, decreased expression of both ERK2 and Akt did not have an additive or synergistic effect. This may mean that they are in the same regulatory pathway or other effectors are regulating the expression of VCAM-1 as well.

The down-regulation of ERK2 and Akt in our study significantly $(P<0.05)$ increased VCAM-1 in cells treated with both insulin and TNFa: a possible scenario in the atherosclerotic vasculature. Kinases are part of common, intracellular pathways that appear to "crosstalk" within the cell [17]. The concert of these signals may keep downstream events in check, whereas their pertubations may cause unbeknownst effects.

Inflammation is associated with many diseases such as obesity, cancer, autoimmunity and atherosclerosis [18-22]. The essential cellular events that initiate the inflammatory process in the vasculature are extracellular signals. They in turn act on cell surface receptors and instigate intracellular signaling via the kinases. It is these kinases that are instrumental in upregulating the inflammatory markers such as VCAM-1 [23, 24].

Interestingly, other studies have established that proinflammatory cytokines such as IL-1 $\beta$ are upregulated by VCAM-1 expression [25]. It will be very interesting to determine if TNF $\alpha$ is upregulated by VCAM-1 at the monocyte as well. If this does occur a positive feedforward mechanism is possible.

Here we report that not only did insulin and TNF $\alpha$ stimulate the expression of VCAM-1, but also that drecreased expression of ERK2 and Akt resulted in increased expression of VCAM-1 at the total protein and cell surface level. Our future studies will evaluate the expression of VCAM-1 in the presence of other inhibitory RNAs; in particular, p38 and JNK. Both p38 and JNK have been shown to be involved with inflammation. Obviously, the knock-down of these two kinases and their effects on VCAM-1 expression will be very interesting and will add to the "cross-talk" picture of the cell. These current and future studies will benefit strategies that will assist in decreasing the inflammatory effects of atherosclerosis in the vasculature.

\section{Conclusions}

Insulin and TNF $\alpha$ increased VCAM-1 expression in RAEC. Yet, RAEC transfected with shAkt and shERK2, which cause decreased expression of Akt and ERK2, initiated increases in total and cell surface VCAM-1 protein in the presence of insulin and/or TNF $\alpha$ greater that seen in mock transfected cells.

\section{Competing interests}

The authors declare that they have no competing interests.

\section{Authors' contributions}

GBP Western blot analysis, flow cytometry analysis and statistical analysis. MT Western blot analysis. NB Western blot analysis. MLG preparation and submission of manuscript. All authors read and approved the final manuscript.

\section{Acknowledgements}

This work was supported by the Research Service of the Department of Veterans Affairs (to M.L.G.), in which Dr. Goalstone is a recipient of a VA Merit Review Award. We would like to thank Ron Bouchard for his excellent work in confocal microscopy and Harsh Pratap for his beneficial assistance with our flow cytometry measurements. The flow cytometer is equipment associated with the Mucosal and Vaccine Research Colorado (MAVRC).

Received: 18 August 2015 Accepted: 19 February 2016

Published online: 25 February 2016

\section{References}

1. Ginsberg HN. Insulin resistance and cardiovascular disease. J Clin Invest. 2000:106:453-8.

2. Breuss JM, Cejna M, Bergmeister H, et al. Activation of nuclear factor-kappa B significantly contributes to lumen loss in a rabbit iliac artery balloon angioplasty model. Circulation. 2002;105:633-8.

3. Reddy KJ, Singh M, Bangit JR, Batsell RR. The role of insulin resistance in the pathogenesis of atherosclerotic cardiovascular disease: an updated review. J Cardiovasc Med (Hagerstown). 2010;11:633-47.

4. Lu H, Raptis M, Black E, Stan M, Amar S, Graves DT. Influence of diabetes on the exacerbation of an inflammatory response in cardiovascular tissue. Endocrinology. 2004;145:4934-9.

5. Walpola PL, Gotlieb Al, Cybulsky MI, Langille BL. Expression of ICAM-1 and VCAM-1 and monocyte adherence in arteries exposed to altered shear stress. Arterioscler Thromb Vasc Biol. 1995;15:2-10.

6. Okouchi M, Okayama N, Imai S, et al. High insulin enhances neutrophil transendothelial migration through increasing surface expression of platelet endothelial cell adhesion molecule-1 via activation of mitogen activated protein kinase. Diabetologia. 2002;45:1449-56.

7. Okouchi M, Okayama N, Shimizu M, Omi H, Fukutomi T, Itoh M. High insulin exacerbates neutrophil-endothelial cell adhesion through endothelial surface expression of intercellular adhesion molecule-1 via activation of protein kinase $\mathrm{C}$ and mitogen-activated protein kinase. Diabetologia. 2002:45:556-9.

8. Madonna R, Pandolfi A, Massaro M, Consoli A, De Caterina R. Insulin enhances vascular cell adhesion molecule-1 expression in human cultured endothelial cells through a pro-atherogenic pathway mediated by p38 mitogen-activated protein-kinase. Diabetologia. 2004;47:532-6.

9. Jiang B, Xu S, Hou X, Pimentel DR, Brecher P, Cohen RA. Temporal control of NF-kappaB activation by ERK differentially regulates interleukin-1 betainduced gene expression. J Biol Chem. 2004:279:1323-9.

10. Wang CC, Lin WN, Lee CW, et al. Involvement of p42/p44 MAPK, p38 MAPK, JNK, and NF-kappaB in IL-1 beta-induced VCAM-1 expression in human tracheal smooth muscle cells. Am J Physiol Lung Cell Mol Physiol. 2005;288: L227-37.

11. Mackesy DZ, Goalstone ML. Extracellular signal-regulated kinase-5: Novel mediator of insulin and tumor necrosis factor alpha-stimulated vascular cell adhesion molecule-1 expression in vascular cells. J Diabetes. 2014;6:595-602.

12. Chang LKarin M. Mammalian MAP kinase signalling cascades. Nature. 2001:410:37-40.

13. Dinev D, Jordan BW, Neufeld B, et al. Extracellular signal regulated kinase 5 (ERK5) is required for the differentiation of muscle cells. EMBO Rep. 2001;2:829-34.

14. Olson EN. Undermining the endothelium by ablation of MAPK-MEF2 signaling. J Clin Invest. 2004;113:1110-2.

15. de la Monte SM, Wands JR. Review of insulin and insulin-like growth factor expression, signaling, and malfunction in the central nervous system: relevance to Alzheimer's disease. J Alzheimers Dis. 2005;7:45-61.

16. Barks JL, McQuillan JJ, lademarco MF. TNF-alpha and IL-4 synergistically increase vascular cell adhesion molecule-1 expression in cultured vascular smooth muscle cells. J Immunol. 1997:159:4532-8.

17. Tanabe Y, Koga M, Saito M, Matsunaga Y, Nakayama K. Inhibition of adipocyte differentiation by mechanical stretching through ERK-mediated downregulation of PPARgamma2. J Cell Sci. 2004;117:3605-14. 
18. Odegaard JI, Chawla A. Pleiotropic actions of insulin resistance and inflammation in metabolic homeostasis. Science. 2013;339:172-7.

19. Han MS, Jung DY, Morel C, et al. JNK expression by macrophages promotes obesity-induced insulin resistance and inflammation. Science. 2013;339:218-22.

20. Erusan RR, Nalini D, Ganesan M, Malathi R. Correlation between Obesity and Inflammation in Cardiovascular Diseases - Evaluation of Leptin and Inflammatory Cytokines. Open J Endoc Metab Dis. 2012;2:7-15.

21. Beishline KAzizkhan-Clifford J. Sp1 and the "Hallmarks of Cancer". FEBS J. 2014; 282:224-58

22. Lee C, Woo Y, Wang Y, Yeung C, Xu A, Lam K. Obesity, adipokines and cancer: An update. Clin Endocrinol (Oxf). 2014;(83):147-56.

23. Habashi JP, Doyle JJ, Holm TM, et al. Angiotensin II type 2 receptor signaling attenuates aortic aneurysm in mice through ERK antagonism. Science. 2011:332:361-5.

24. Nakashima $H$, Suzuki $H$, Ohtsu $H$, et al. Angiotensin II regulates vascular and endothelial dysfunction: recent topics of Angiotensin II type-1 receptor signaling in the vasculature. Curr Vasc Pharmacol. 2006;4:67-78.

25. Zohlnhofer D, Brand K, Schipek K, Pogatsa-Murray G, Schomig A, Neumann FJ. Adhesion of monocyte very late antigen-4 to endothelial vascular cell adhesion molecule-1 induces interleukin-1 beta-dependent expression of interleukin-6 in endothelial cells. Arterioscler Thromb Vasc Biol. 2000;20:353-9.

\section{Submit your next manuscript to BioMed Central} and we will help you at every step:

- We accept pre-submission inquiries

- Our selector tool helps you to find the most relevant journal

- We provide round the clock customer support

- Convenient online submission

- Thorough peer review

- Inclusion in PubMed and all major indexing services

- Maximum visibility for your research

Submit your manuscript at www.biomedcentral.com/submit

) Biomed Central 